\title{
PLASMA HYDROGENATION OF HYDROTHERMALLY GROWN ZNO MICROPODS
}

\author{
'Zdeněk REMEŠ, ²Kateřina AUBRECHTOVÁ DRAGOUNOVÁ, ${ }^{3} J u l i a$ MIČOVÁ \\ ${ }^{1}$ FZÚ- Institute of Physics, Czech Academy of Sciences, Praha, Czech Republic,EU, remes@fzu.cz \\ ${ }^{2}$ FZÚ- Institute of Physics, Czech Academy of Sciences, Praha, Czech Republic, EU, dragounova@fzu.cz \\ 3Institute of Chemistry, Slovak Academy of Sciences, Bratislava, Slovakia, EU, chemjumi@savba.sk
}

https://doi.org/10.37904/nanocon.2020.3777

\begin{abstract}
The hydrothermally grown $\mathrm{ZnO}$ micropods have been placed on grounded stainless-steel holder and exposed to an inductively coupled plasma (ICP) monitored in-situ by optical emission spectroscopy (OES). OES shows the immediate release of oxygen during $\mathrm{Ar}$ ion bombardment. The prolonged exposure to hydrogen plasma leads to deterioration of the optical properties as well. The exposure, if power and hydrogen pressure have been optimized to enhance UV-photoluminescence peak at the wavelength $384 \mathrm{~nm}$ related to surface bounded excitons and reduce the defect-related photoluminescence in red spectral range. The strong UV photoluminescence appears just after 1 minute of plasma hydrogenation in a radio frequency plasma discharge with power density $40 \mathrm{~W} / \mathrm{dm}^{3}$ and hydrogen pressure $17 \mathrm{~Pa}$.
\end{abstract}

Keywords: $\mathrm{ZnO}$, micropods, inductively coupled plasma, optical emission spectroscopy, photoluminescence spectroscopy, Raman spectroscopy, infrared absorption spectroscopy

\section{INTRODUCTION}

The zinc oxide $(\mathrm{ZnO})$ crystallite powder is a low cost, environmentally friendly, wide band gap semiconducting material with unique optical properties and variety of nanoparticle (NPs) morphologies [1]. Due to the high surface-to-volume ratio and related size effects, ZnO NPs are used in many applications for energy conversion, photocatalytic wastewater treatment, electrochemical energy storage or sensing. Recently, we have applied a hydrothermal chemical synthesis as a low-cost technology for mass-production of $\mathrm{ZnO}$ hedgehog-like crystallite powder [2], studied their crystal quality [3] and shown that the age of precursors plays a significant role in defect formation [4]. The photoluminescence spectra (PLS) show a strong near UV emission peak related to bound exciton complexes assigned to surface states [5]. The strong correlation between surface defects and the exciton emission has been also observed in hydrothermally synthetized $\mathrm{ZnO}$ micropods [6].

Particularly important are electrically conductive ZnO NPs with the interstitial hydrogen acting as a donor [7]. Two-step annealing in air at around $1000{ }^{\circ} \mathrm{C}$ followed by annealing in hydrogen atmosphere at $800{ }^{\circ} \mathrm{C}$ has been demonstrated to significantly enhance UV-photoluminescence of ZnO NPs for highly efficient subnanosecond fast scintillators $[8,9]$. The inductively coupled plasma (ICP) offers an alternative way of cold hydrogenation [10]. We have already shown that the cold hydrogenation in low pressure hydrogen plasma is an effective way to significantly enhance the electrical conductivity and exciton-related emission in $\mathrm{ZnO}$ thin films prepared by reactive magnetron sputtering on fused silica glass substrates [11]. In this paper we optimize the plasma hydrogenation of $\mathrm{ZnO}$ micropods placed on grounded stainless-steel sample holder. 


\section{EXPERIMENTAL}

\subsection{Hydrothermal growth}

$\mathrm{ZnO}$ micropods were the hydrothermally synthesized in aqueous solution containing equimolar ( $25 \mathrm{mM})$ amount of zinc nitrate hexahydrate p.a. $\left(\mathrm{Zn}\left(\mathrm{NO}_{3}\right)_{2} \cdot 6 \mathrm{H}_{2} \mathrm{O}\right)$ and hexamethylenetetramine $(\mathrm{HMTA})\left(\mathrm{C}_{6} \mathrm{H}_{12} \mathrm{~N}_{4}\right)$ p.a. Both chemicals were purchased from Slavus and used without further purification. Deionized water was purified with a system So Safe Water Technologies, having a conductivity $0.20 \mu \mathrm{S} \cdot \mathrm{cm}^{-1}\left(25^{\circ} \mathrm{C}\right)$. The reaction mixture was heated at $90^{\circ} \mathrm{C}$ for $3 \mathrm{~h}$. The precursor salt residues were removed from the sample by washing 5-times with deionized water followed by centrifugation at $10000 \mathrm{rpm}$ (RCF: $11510 \mathrm{~g}$ ) for $15 \mathrm{~min}$. Finally, the $\mathrm{ZnO}$ powder was dried by lyophilization. Scanning electron microscopy (SEM) images show ZnO micropods with hexagonal shape, the length up to $10 \mu \mathrm{m}$ and the diameter about $0.5 \mu \mathrm{m}$, see Figure 1.
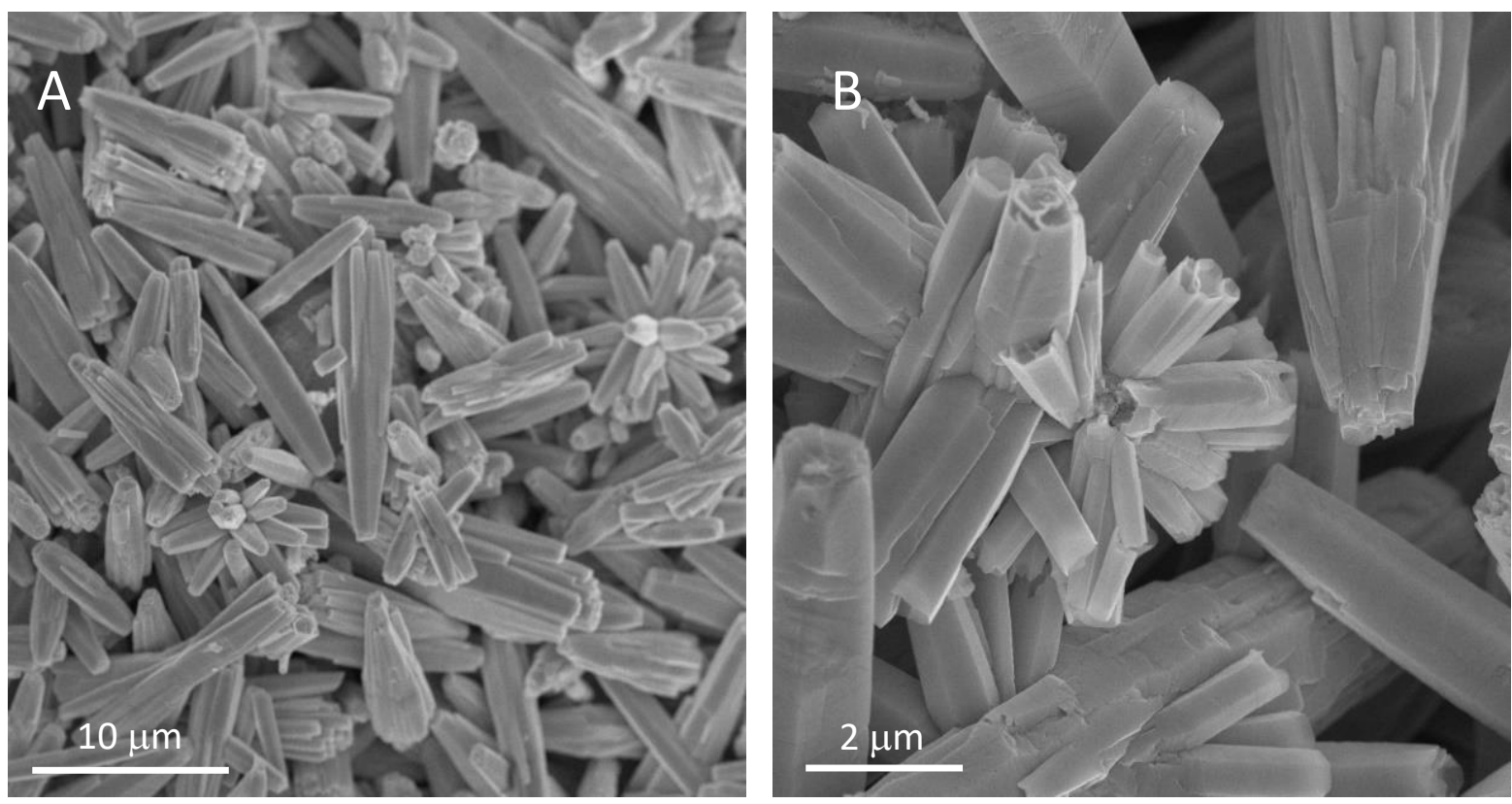

Figure 1 SEM images of hydrothermally grown ZnO micropods taken by MAIA3 (TESCAN) using the electron beam energy $5 \mathrm{keV}(\mathrm{A})$ and $20 \mathrm{keV}(\mathrm{B})$

\subsection{Plasma treatment}

The plasma hydrogenation has been done in a prototype inductively coupled plasma (ICP) reactor currently being developed in a cooperation with the Czech company SVCS Process Innovation, s. r. o. (Valašské Meziríčí, Czech Republic). The quartz reactor of the active volume 5 liters operates at radio frequency (rf) 13.56 $\mathrm{MHz}$ with maximum discharge power up to $300 \mathrm{~W}$ corresponding to the power density $60 \mathrm{~W} / \mathrm{dm}^{3}$. The setup is evacuated with the Agilent Triscroll 300 dry vacuum pump protected with the ISO Flange Vacuum Filter (CSL-843-NW25). A $5 \mathrm{mg}$ powder sample mechanically pressed into a round pellet with the diameter $3 \mathrm{~mm}$ was placed in the middle of the chamber on a grounded stainless-steel holder. The holder grounding is a recent improvement over the previously used ungrounded quartz holder. Prior the plasma treatment, the chamber, valves, flowmeters, gauges and all gas inlet and outlet tubes were evacuated to residual pressure below $0.1 \mathrm{~Pa}$ and flushed $5 \mathrm{~min}$ by $20 \mathrm{sccm}$ flow of process gases to reduce residual gas contamination. The pellets were exposed for $30 \mathrm{~s}$ to Ar plasma (Ar purity 99.998\%, if power $100 \mathrm{~W}$, Ar flow $5 \mathrm{sccm}$, pressure $16 \mathrm{~Pa})$ followed by up to 20 min of hydrogen plasma exposure $\left(\mathrm{H}_{2}\right.$ purity $99.999 \%$, rf power $200 \mathrm{~W}, \mathrm{H}_{2}$ flow $20 \mathrm{sccm}$, pressure $17 \mathrm{~Pa}$ ). The originally white $\mathrm{ZnO}$ pellets became darker after prolonged plasma hydrogenation, see Figure 2. 


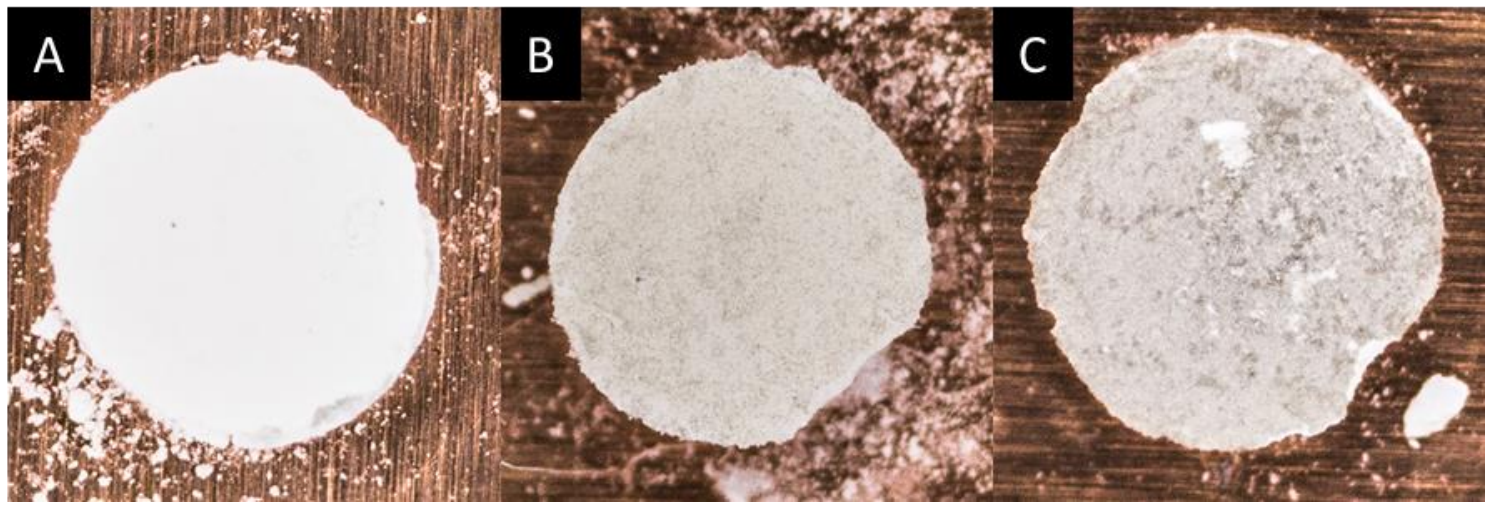

Figure 2 Optical images of $\mathrm{ZnO}$ pellets (diameter $3 \mathrm{~mm}$ ) after $2 \mathrm{~min}(\mathrm{~A}), 10 \mathrm{~min}(\mathrm{~B})$ and $20 \mathrm{~min}(\mathrm{C})$ plasma hydrogenation taken with a macro objective (Lumix G Macro $30 \mathrm{~mm}$, F2.8 ASPH).

\subsection{Characterization}

Optical emission spectra (OES) were measured in 400-1000 nm spectral range with $1 \mathrm{~nm}$ spectral resolution by spectrally calibrated fiber coupled CCD spectrometer (B\&W Tek BTC112E) focused in the middle of the quartz chamber. Dark spectra were subtracted. The integration time varied from $5 \mathrm{~ms}$ for Ar plasma to $200 \mathrm{~ms}$ for $\mathrm{H}_{2}$ plasma. Each spectrum was averaged 10 times.

Raman spectra were recorded in backscatter geometry using a micro-spectroscopic Renishaw InVia Reflex Raman setup with integrated optical microscope and a charge-coupled device (CCD) camera. For excitation, a 442- nm-line of HeCd excitation laser. Other parameters were 100× Olympus objective, $65 \mu \mathrm{m}$ slits, and a grating of 2400 grooves $/ \mathrm{mm}$.

The attenuated total reflectance (ATR) infrared absorption spectra were detected in $590-5000 \mathrm{~cm}^{-1}$ spectral range and $4 \mathrm{~cm}^{-1}$ resolution using Nicolet ${ }^{\mathrm{TM}}$ iS50 FTIR Spectrometer (Thermo Fisher Scientific) with build-in diamond ATR prism, $\mathrm{KBr}$ beam splitter and cooled DTGS detector. The ATR spectra were first normalized to zero at $5000 \mathrm{~cm}^{-1}$ and then recalculated with the build-in advanced ATR correction for diamond prism.

PLS were measured at room temperature in the $360-780 \mathrm{~nm}$ spectral range with $2 \mathrm{~nm}$ spectral resolution using the $100 \mathrm{~mW} / \mathrm{cm}^{2}$ UV photo-excitation provided by a focused $340 \mathrm{~nm}$ LED equipped with a narrow band-pass filter and operating in square pulse regime at frequency $307 \mathrm{~Hz}$. The emitted and scattered light was collected and focused onto the $1 \mathrm{~mm}$ wide input slit of double gratings SPEX 1672 monochromator equipped with Peltier cooled multi-dynode multi-alkali red sensitive photomultiplier (PMT) to be measured at each wavelength independently by a lock-in amplifier synchronized with excitation frequency. The spectra above $600 \mathrm{~nm}$ were measured with a long pass filter placed in front of the monochromator. The whole setup was spectrally calibrated with Oriel \#63358 Quartz Tungsten halogen lamp and converted from wavelength to energy scale for quantitative analysis [12].

\section{RESULTS AND DISCUSSION}

The OES spectra clearly show the presence of atomic oxygen in Ar plasma as demonstrated by the presence of oxygen-related emission peaks at 777 and $845 \mathrm{~nm}$ [13], see Figure 3. Since no nitrogen related peaks are observed, oxygen cannot originate from ambient atmosphere leak, but from $\mathrm{ZnO}$ powder. In pure Ar plasma the intensity of O-peaks is low and may be explained by contamination from previously processed $\mathrm{ZnO}$. The intensity of O-peaks increases by an order of magnitude when a pellet is placed into the chamber while the plasma visually change color from brownish to white. Thus, OES proves the heavy ion bombardment of the sample placed on grounded holder. Since only the Balmer series lines were observed in hydrogen plasma at 656, 486 and $434 \mathrm{~nm}$ and no O-peaks, we conclude that $\mathrm{ZnO}$ was scattered by heavy Ar ions but not by light $\mathrm{H}$ ions. 

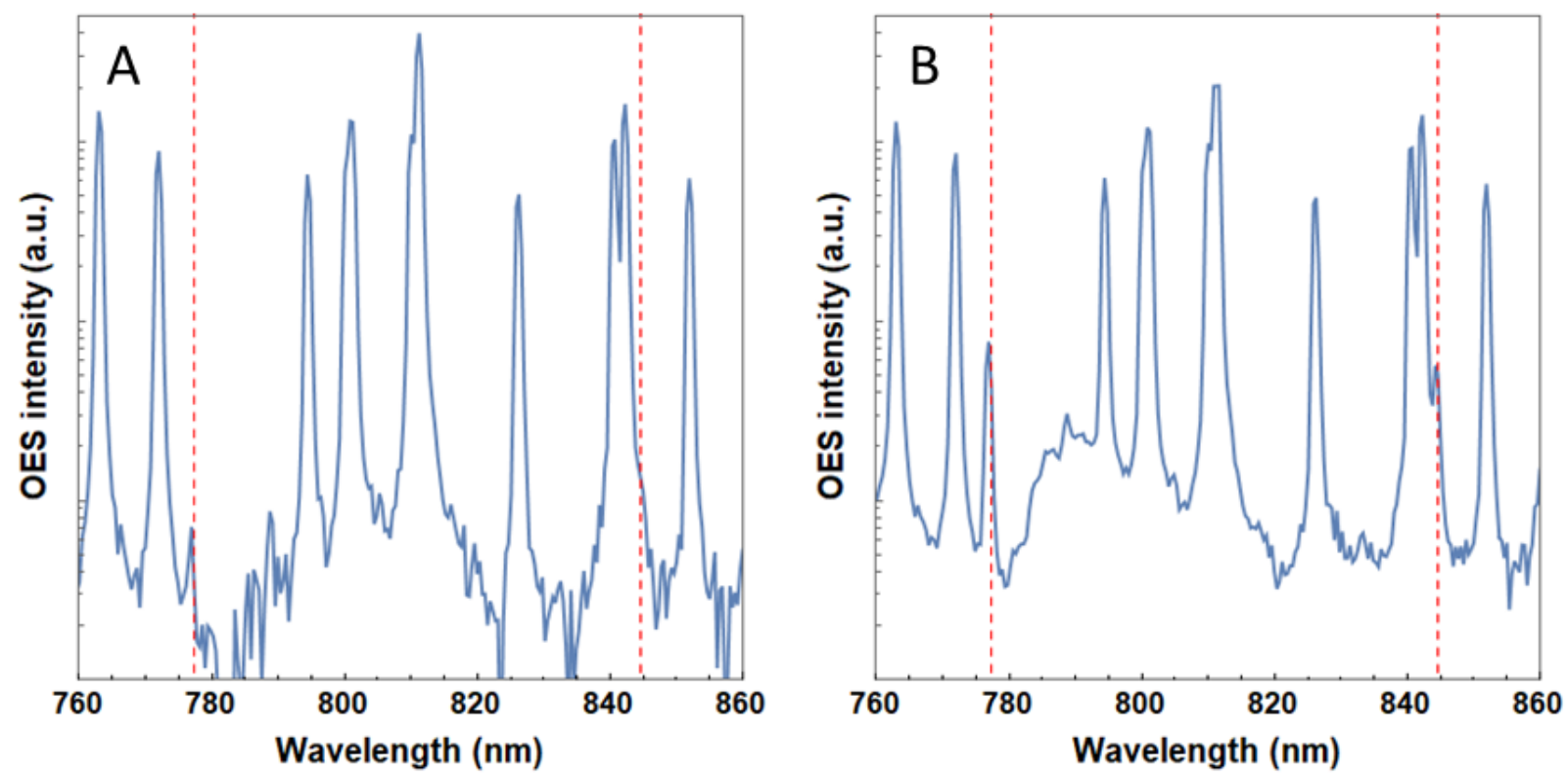

Figure 3 The semilogarithmic optical emission spectra of Ar plasma without $(A)$ and with (B) ZnO pellet placed onto grounded stainless steel holder in the ICP reactor. The position of oxygen emission lines at 777 and $845 \mathrm{~nm}$ is indicated by dashed red lines.

Figure 4 (A) shows Raman spectra of plasma hydrogenated $\mathrm{ZnO}$ micropods, where origin of peaks is also depicted. Nonpolar phonon mode $\mathrm{E}_{2}$ (Low) expected at $100 \mathrm{~cm}^{-1}$ is associated with $\mathrm{Zn}$ sublattice, but it was out of the measured spectral range. Nonpolar phonon mode $E_{2}$ (High) associated with oxygen sublattice is observed at $440 \mathrm{~cm}^{-1}$ [14]. The sharp, strong and dominant Raman peak is significantly amplified after hydrogen plasma, but for 10-minutes hydrogenation and longer its intensity decreases again. Same as increasing intensity of the dominant peak, broad sideband at low-frequency side also increases. This broad band with maximum about $420 \mathrm{~cm}^{-1}$ is attributed to $E_{1}(\mathrm{TO})$ mode commonly seen in nano- and microstructures. The peaks at 336 and $540 \mathrm{~cm}^{-1}$ correspond to the sum and differential wavenumbers $\mathrm{E}_{2}(\mathrm{High})$ $\pm E_{2}$ (Low) and $A_{1}(L O)$, respectively [15]. The peak at $581 \mathrm{~cm}^{-1}$ has been assigned to the $\mathrm{ZnO}$ mode $\mathrm{E}_{1}(\mathrm{LO})$ [16]. This mode should be inactive but it may appear as a local mode due to the disturbance of the crystal symmetry resulting from the presence of oxygen vacancies and zinc interstitials. Thus, the presence of this mode suggests that the plasma treatment increases the concentration of point defects. Moreover, from spectra normalized on the value of $E_{2}$ (High) peak, see Figure 4(B), could be seen that for hydrogenation time up to 5 minutes, the spectra are very similar. After longer hydrogenation, the height of the $E_{1}(\mathrm{LO})$ peak decreases. In spectra, small band near $660 \mathrm{~cm}^{-1}$ and $1150 \mathrm{~cm}^{-1}$ correspond to multiphonon processes and peak near 1100 $\mathrm{cm}^{-1}$ to combination of acoustic modes with symmetry $A_{1}$ and $E_{2}$.

ATR-FTIR is a sensitive probe of free carriers in $\mathrm{ZnO}$. It has been shown that the room-temperature exposure of polycrystalline $\mathrm{ZnO}$ films to $\mathrm{H}_{2}$ plasma rapidly increases carrier density [17]. Figure 5 shows the infrared absorbance spectra of plasma hydrogenated $\mathrm{ZnO}$ micropods depending on the expose to hydrogen plasma. Free carrier absorption dominates infrared absorbance spectra already in the unhydrogenated sample with further increase after plasma hydrogenation. The room temperature plasma hydrogenation had been sufficient to induce significant surface conductivity changes after 1 minute expose. It should be noted that the absorbance has been somewhat arbitrary normalized to zero at $5000 \mathrm{~cm}^{-1}$ and that the signal-to-noise ratio is poor in the spectral range $2000-2300 \mathrm{~cm}^{-1}$ where diamond ATR prism heavily absorbs. 


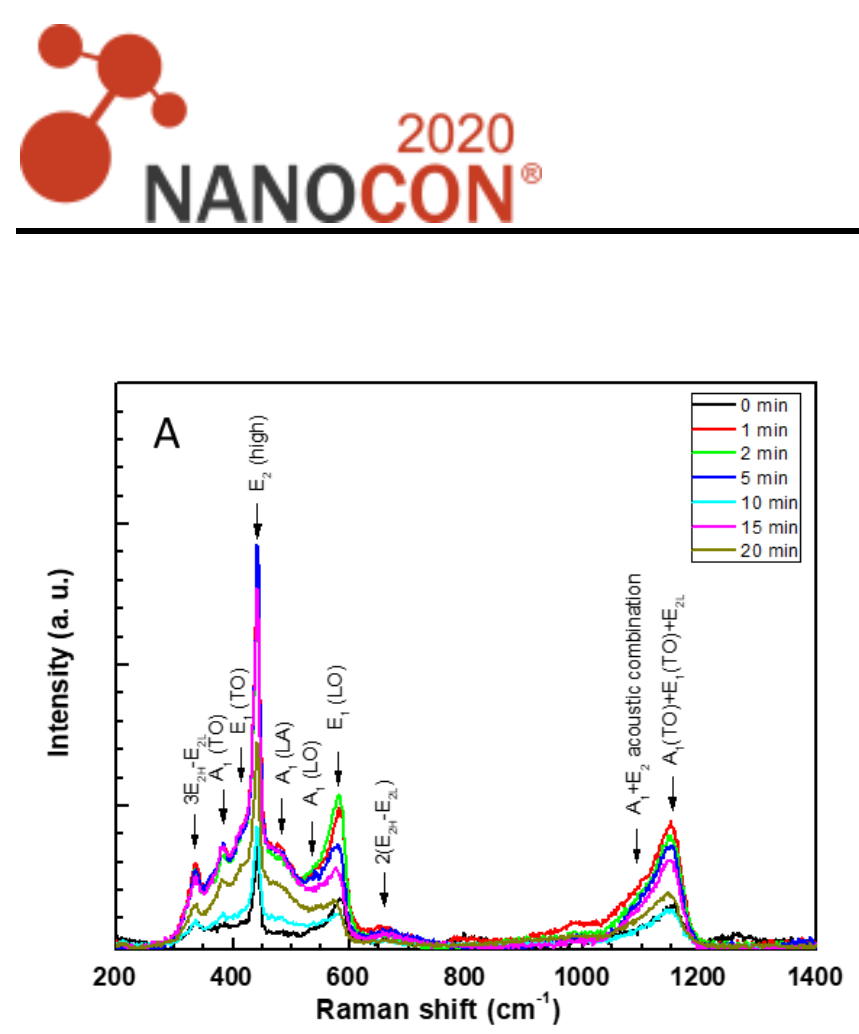

Figure 4 Raman spectra (A) and normalized Raman spectra (B) of plasma hydrogenated ZnO micropods depending on the expose to hydrogen plasma. The excitation wavelength was $442 \mathrm{~nm}$ (blue laser)

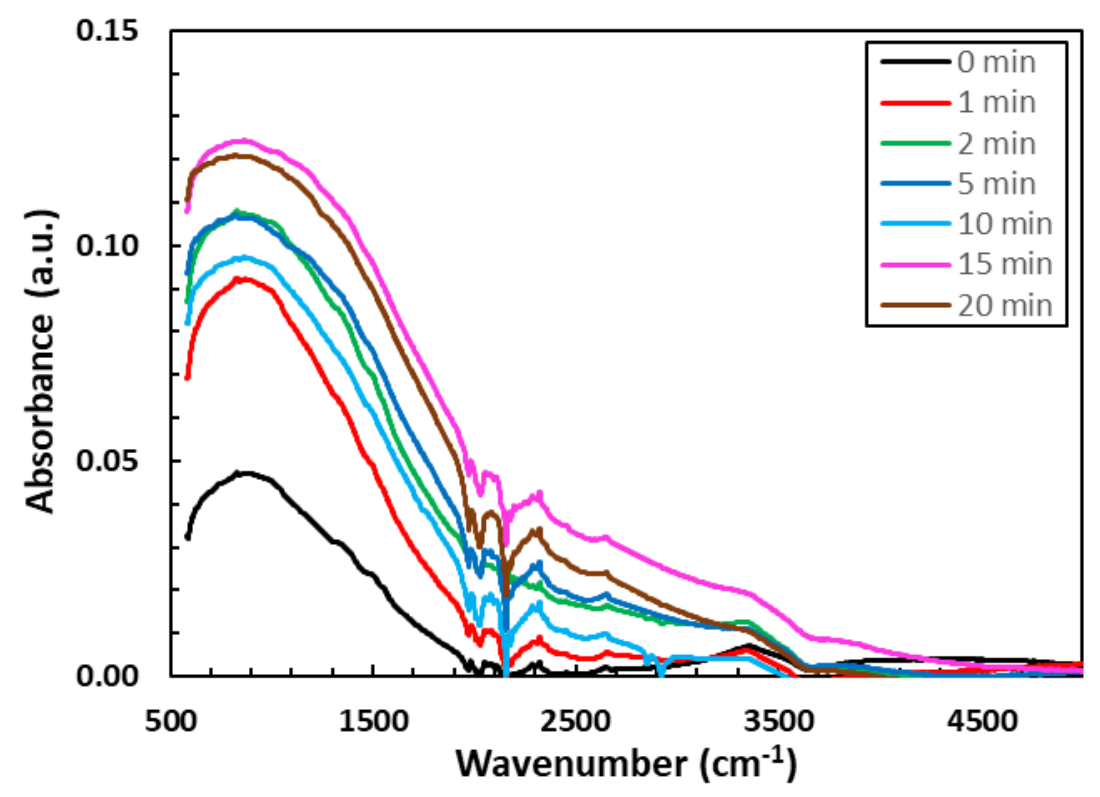

Figure 5 Infrared absorbance spectra of plasma hydrogenated $\mathrm{ZnO}$ micropods depending on the expose to hydrogen plasma measured by ATR FTIR and normalized to zero at $5000 \mathrm{~cm}^{-1}$.

The PLS in Figure 6 shows that the strong exciton photoluminescence at $3.23 \mathrm{eV}(384 \mathrm{~nm})$ appears already after 1 min plasma hydrogenation at $200 \mathrm{~W}$. This suggest that the bound exciton complexes are assigned to surface states. Furthermore, the hydrogen plasma suppresses the defect related broad PLS band centered in red spectra range around $2 \mathrm{eV}$. It takes about $10 \mathrm{~min}$ to suppress this band by an order of magnitude. Therefore, we suggest 5 min plasma hydrogenation as an optimal process time. This is significantly shorter time then 30 min previously used in plasma hydrogenation with ungrounded quartz holder. 

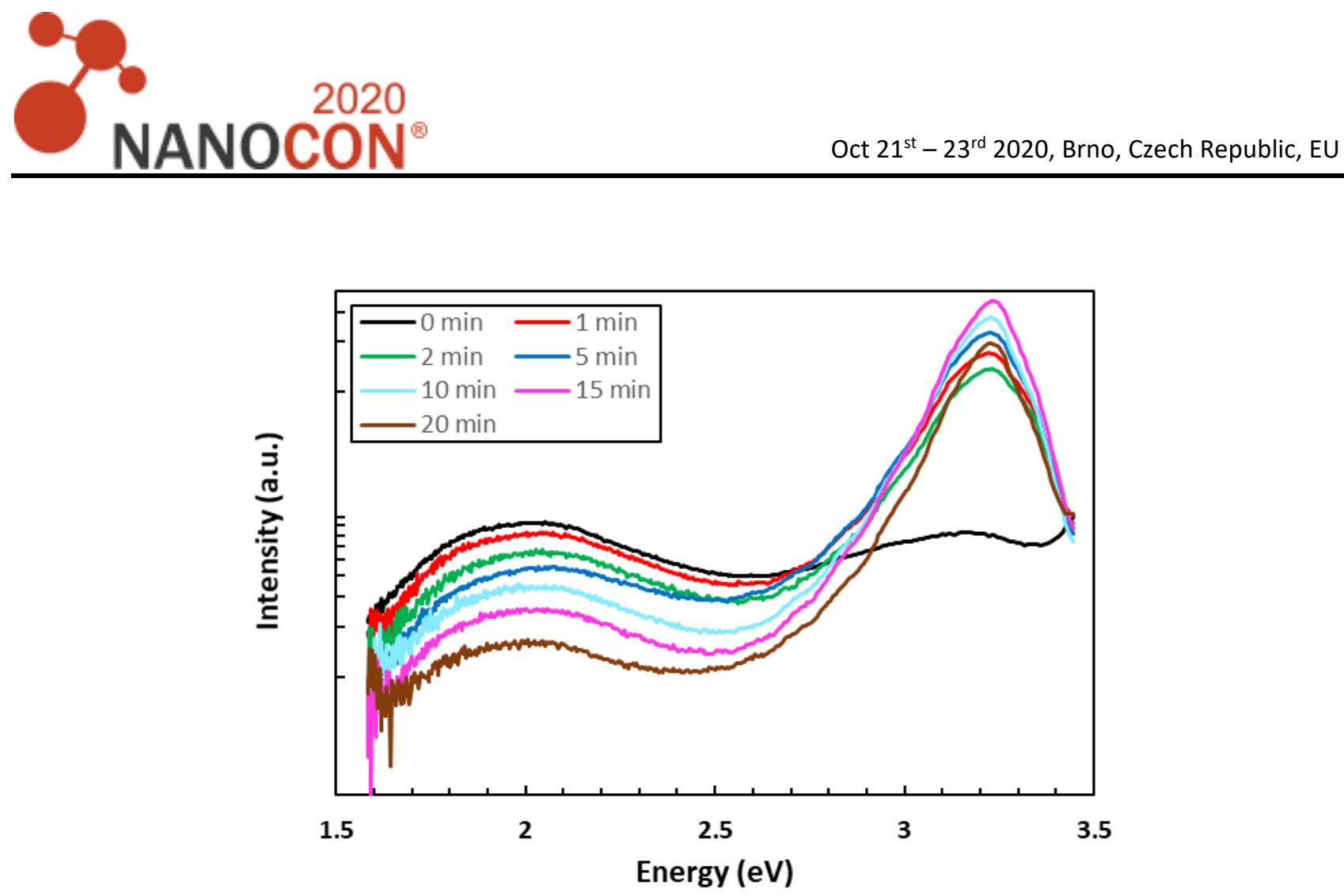

Figure 6 The room temperature PLS of plasma hydrogenated $\mathrm{ZnO}$ micropods in semilogarithmic intensity scale depending on the expose to hydrogen plasma. The excitation energy was $3.65 \mathrm{eV}$.

\section{CONCLUSION}

Ar plasma sputters $\mathrm{ZnO}$ placed on a grounded stainless-steel holder in an ICP reactor. Therefore, the Ar cleaning process needs to be taken only for a short time with a relatively low if power. The strong UV photoluminescence appears just after a minute of plasma hydrogenation of $\mathrm{ZnO}$ micropods which is a strong evidence that the bound exciton complexes are assigned to surface states. On the other hand, the reduction of red photoluminescence needs prolonged time suggesting that the red photoluminescence is related to bulk defect states. The optimal time for plasma hydrogenation at the rf power density $40 \mathrm{~W} / \mathrm{dm}^{3}$ and hydrogen pressure $17 \mathrm{~Pa}$ seems to be just a few minutes.

\section{ACKNOWLEDGEMENTS}

This work was supported by the Czech Science Foundation project 19-02858J, by the Operational Programme Research, Development and Education project SOLID21 -

CZ.02.1.01/0.0/0.0/16_019/0000760 and by the Scientific Grant Agency of Ministry of Education, Science, Research and Sport of Slovak Republic and Slovak Academy of Sciences (VEGA 2/0157/20). Special thanks to Ing. Rayisa Yatskiv from the Institute of Physics of the Czech Academy of Sciences for SEM.

\section{REFERENCES}

[1] KLINGSHIRN, C. F. (ed.). Zinc Oxide: from fundamental properties towards novel applications. Heidelberg; London : Springer, 2010. Springer series in materials science, 120. ISBN 978-3-642-10576-0. QD181.Z6 Z56 2010.

[2] CHANG, Yu-Ying, REMES, Zdenek, MICOVA, Julia. Mass production of hydrogenated ZnO nanorods. In : NANOCON 2019 - Conference Proceedings [online]. Hotel Voronez I, Brno, Czech Republic:Tanger Ltd., 2020. pp. 221-225. [Accessed 11 May 2020]. ISBN 978-80-87294-95-6. Available from: https://www.confer.cz/nanocon2019-8680-chang-mass-production-of-hydrogenated-zno-nanorods.pdf

[3] MIČOVÁ, Júlia, BURYI, Maksym, ŠIMEK, Daniel, DRAHOKOUPIL, Jan, NEYKOVA, Neda, CHANG, Yu-Ying, REMEŠ, Zdeněk, POP-GEORGIEVSKI, Ognen, SVOBODA, Jan, IM, Chan. Synthesis of zinc oxide 
nanostructures and comparison of their crystal quality. Applied Surface Science. December 2018, vol. 461, pp. 190-195. DOI: 10.1016/j.apsusc.2018.05.176.

[4] BURYI, M., BABIN, V., CHANG, Y.-Y., REMEŠ, Z., MIČOVÁ, J., ŠIMEK, D. Influence of precursor age on defect states in ZnO nanorods. Applied Surface Science. September 2020, vol. 525, p. 146448. DOI: 10.1016/j.apsusc.2020.146448.

[5] FALLERT, Johannes, HAUSCHILD, Robert, STELZL, Felix, URBAN, Alex, WISSINGER, Markus, ZHOU, Huijuan, KLINGSHIRN, Claus, KALT, Heinz. Surface-state related luminescence in $\mathrm{ZnO}$ nanocrystals. Journal of Applied Physics. April 2007, vol. 101, no. 7, p. 073506. DOI: 10.1063/1.2718290.

[6] GOKARNA, Anisha, AAD, Roy, ZHOU, Junze, NOMENYO, Komla, LUSSON, Alain, MISKA, Patrice, LERONDEL, Gilles. On the origin of the enhancement of defect related visible emission in annealed $\mathrm{ZnO}$ micropods. Journal of Applied Physics. 14 October 2019, vol. 126, no. 14, p. 145104. DOI: 10.1063/1.5111184.

[7] VAN DE WALLE, Chris G., NEUGEBAUER, J. Universal alignment of hydrogen levels in semiconductors, insulators and solutions. Nature. June 2003, vol. 423, no. 6940, pp. 626-628. DOI: 10.1038/nature01665.

[8] BOURRET-COURCHESNE, E.D., DERENZO, S.E., WEBER, M.J. Development of ZnO:Ga as an ultra-fast scintillator. Nuclear Instruments and Methods in Physics Research Section A: Accelerators, Spectrometers, Detectors and Associated Equipment. April 2009, vol. 601, no. 3, pp. 358-363. DOI: 10.1016/j.nima.2008.12.206.

[9] [PROCHÁZKOVÁ, Lenka, GBUR, Tomáš, ČUBA, Václav, JARÝ, Vítězslav, NIKL, Martin. Fabrication of highly efficient ZnO nanoscintillators. Optical Materials. September 2015, vol. 47, pp. 67-71. DOI:

10.1016/j.optmat.2015.07.001.

[10] GRILL, Alfred. Cold plasma in material fabrication: from fundamentals to applications. New York: The Institute of Electrical and Electronics Engineers, Inc., 1994. ISBN 0-7803-4714-5.

[11] CHANG, Y.-Y., NEYKOVA, N., STUCHLIK, J., PURKRT, A., REMES, Z. Hydrogen plasma treatment of ZnO thin films. In: NANOCON 2016 - Conference Proceedings. Ostrava, Czech Republic: TANGER Ltd., 2016. pp. 161165. ISBN 978-80-87294-71-0.

[12] MOONEY, Jonathan, KAMBHAMPATI, Patanjali. Get the Basics Right: Jacobian Conversion of Wavelength and Energy Scales for Quantitative Analysis of Emission Spectra. The Journal of Physical Chemistry Letters. 3 October 2013, vol. 4, no. 19, pp. 3316-3318. DOI: 10.1021/jz401508t.

[13] KRAMIDA, Alexander, RALCHENKO, Yuri. NIST Atomic Spectra Database, NIST Standard Reference Database 78 [online]. 1999. National Institute of Standards and Technology. [Accessed 25 August 2020]. Available from: http://www.nist.gov/pml/data/asd.cfm.

[14] YANG, J. H., ZHENG, J. H., ZHAI, H. J., YANG, L. L. Low temperature hydrothermal growth and optical properties of ZnO nanorods. Crystal Research and Technology. January 2009, vol. 44, no. 1, pp. 87-91. DOI: 10.1002/crat.200800294.

[15] CALLEJA, J. M., CARDONA, Manuel. Resonant Raman scattering in ZnO. Physical Review B. 15 October 1977, vol. 16, no. 8, pp. 3753-3761. DOI: 10.1103/PhysRevB.16.3753.

[16] DECREMPS, Fredéric, PELLICER-PORRES, Julio, SAITTA, A. Marco, CHERVIN, Jean-Claude, POLIAN, Alain. High-pressure Raman spectroscopy study of wurtzite ZnO. Physical Review B [online]. 6 February 2002, vol. 65, no. 9. [Accessed 29 February 2020]. DOI: 10.1103/PhysRevB.65.092101. Available from: https://link.aps.org/doi/10.1103/PhysRevB.65.092101.

[17] WOLDEN, C.A., BARNES, T.M., BAXTER, J.B., AYDIL, E.S. Infrared detection of hydrogen-generated free carriers in polycrystalline ZnO thin films. Journal of Applied Physics [online]. 2005, vol. 97, no. 4. DOI: 10.1063/1.1851599. 